\title{
ESTUDIO DE PREFACTIBILIDAD TECNO-ECONÓMICO Y AMBIENTAL PARA LA CONSTRUCCIÓN DEL RELLENO SANITARIO DEL MUNICIPIO DE ESTELÍ
}

Ing. Jimmy Sierra Mercado

Correo: Jimmy.sierra@uni.norte.edu.ni.

Coordinador de Ingeniería Civil

UNI-Norte.
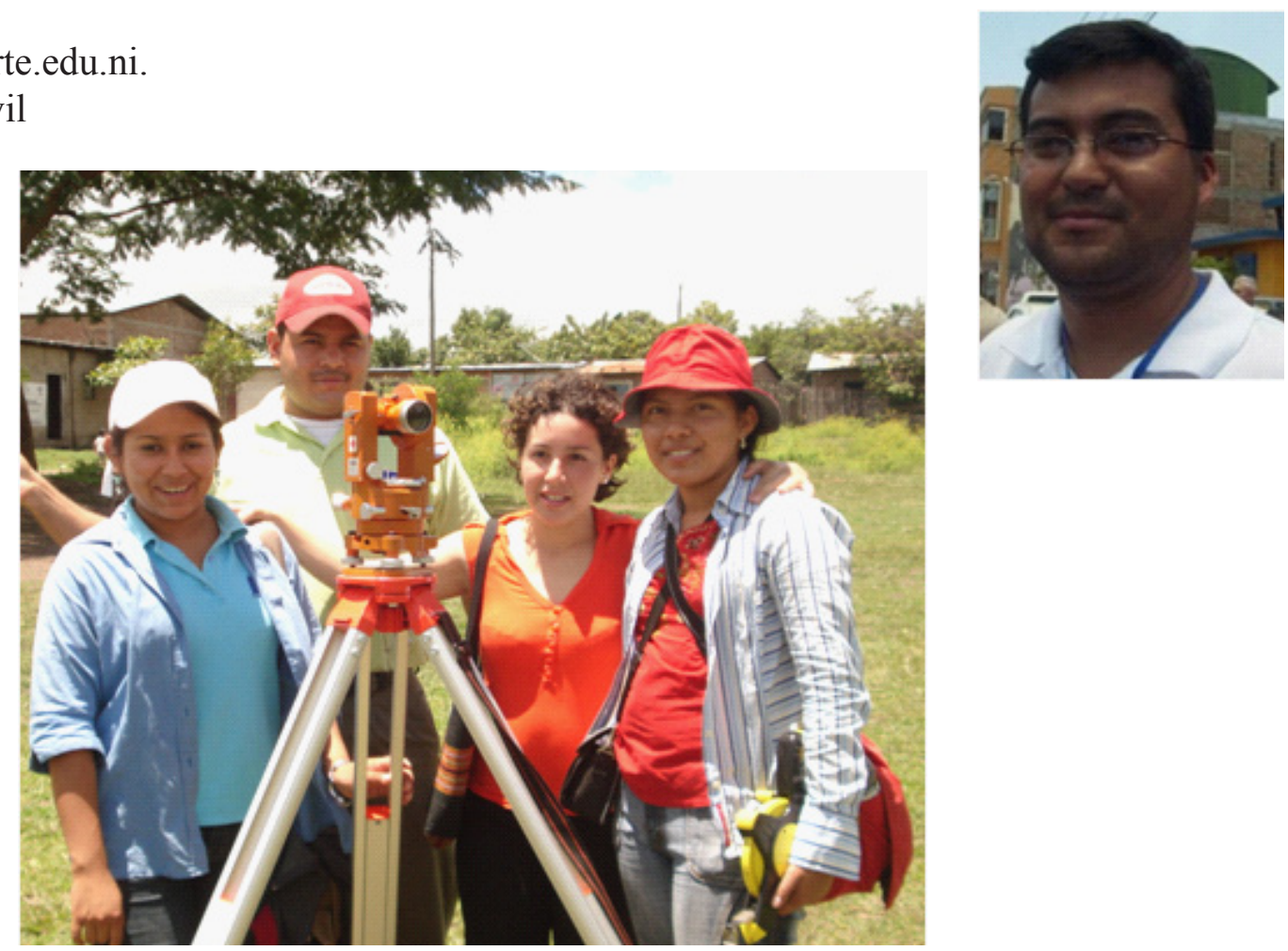

\section{RESUMEN}

El estudio fue realizado en el Municipio de Estelí, donde existe actualmente un vertedero que no presta las condiciones para el tratamiento de los desechos sólidos urbanos. Se debe a que tiene un área de inundación en la parte sur oeste y las trincheras no funcionan adecuadamente. Existe además un riesgo ambiental y tiene una corta vida útil. Esta investigación tiene el propósito de describir las condiciones técnicas, económicas y ambientales para la construcción de un nuevo relleno sanitario municipal que permitirá que la población goce de un ambiente limpio y sano, sin la presencia de basura expuesta al aire libre. Se identifican las diferentes actividades y el estudio de impacto ambiental para el nuevo relleno sanitario del municipio de Estelí, que se encontraría ubicado a $4 \mathrm{~km}$ del perímetro de la ciudad, en la comunidad "La Cruz", donde actualmente existe material selecto para la construcción. La caracterización física de los residuos sólidos que resultó del estudio, arrojó una producción per cápita de $0.42 \mathrm{~kg} / \mathrm{hab} /$ día, que corresponde a una producción diaria de 39.52 toneladas y una densidad de $244.69 \mathrm{~kg} / \mathrm{m} 3$, con un porcentaje de materia orgánica del 63.75\%. Las obras de mayor importancia en la realización de este proyecto: el sistema de captación de los residuos líquidos (lixiviados), recolectados en estructuras (zanjas dentro de la trinchera), cubiertas de material local (piedras de 3", 4" y 5"), la instalación de chimeneas para la evacuación de gases generados en cada trinchera; construcción de un sistema de tratamiento de los lixiviados recolectados; construcción de caseta de control y obras secundarias (cerco perimetral, drenaje de aguas pluviales, etc.). Para mitigar el impacto ambiental que generará la instalación del nuevo relleno, se propone la siembra de árboles alrededor del local. Todo lo expuesto anteriormente, garantizará que el proyecto sea factible desde el punto de vista técnico, económico y ambiental.

Palabras claves: vertedero, desechos sólidos, relleno sanitario, trinchera, lixiviados. 


\section{INTRODUCCION}

El relleno sanitario es un método diseñado para la disposición final de la basura. Consiste en depositar en el suelo los desechos sólidos, los cuales se esparcen y compactan, reduciéndolos al menor volumen posible para que ocupen un área pequeña. Luego se cubren con una capa de tierra y se compactan nuevamente al terminar el día.

El actual "Relleno sanitario" de la ciudad de Estelí, se sitúa a $7 \mathrm{~km}$ del casco urbano $(5 \mathrm{~km}$ de terracería y con algunas pendientes pronunciadas) y fue inaugurado en 1997. Para la instalación de este relleno no se realizaron estudios previos de análisis de suelo y presenta la desventaja que el $50 \%$ del terreno tiene subsuelo rocoso y se dificulta el tratamiento de relleno sanitario. No dispone de zanja perimetral de evacuación de pluviales, ni de lixiviados, no tiene cerco adecuado y carece de una caseta de control. Durante el verano se excavan trincheras y se entierra deficientemente la basura una vez por semana. En el invierno se deposita la basura a cielo abierto, en la parte alta rocosa del vertedero. Los residuos de aserríos y fábricas de tabaco se queman en un área cercana a la entrada, sin ninguna protección anti incendio. Adicional a esto, no hay un estudio de la vida útil del vertedero.

No existe ningún proyecto alternativo al vertedero actual. Es necesario un estudio de factibilidad para una nueva ubicación de un relleno sanitario. De suma importancia para el municipio de Estelí, ya que permitirá dar respuesta a los problemas relacionados con el manejo de los residuos sólidos.

Esta investigación involucra un estudio de pre factibilidad tecno- económico y ambiental para un nuevo relleno sanitario, ubicado en un sitio diferente y bajo las normativas estipuladas para su diseño.

\section{MATERIALES Y MÉTODOS}

La ubicación del sitio para el nuevo relleno sanitario se realizó evaluando las tres alternativas, bajo los criterios del INIFOM y se validó por el método empleado por Lazo, et al. 2003. Se comparó la ubicación del sitio actual, con respecto a la ubicación del nuevo relleno en las comunidades "La Cruz" y "Las Pintadas".
Una vez elegido el sitio para la disposición final de los desechos sólidos se procede al cálculo del área requerida para la construcción del relleno sanitario, basado en el crecimiento de la población, proyectando la vida útil del relleno para 20 años.

Para definir el método constructivo del relleno sanitario se hicieron los estudios de suelos y el levantamiento topográfico. Los parámetros utilizados para el cálculo de lixiviados son la precipitación pluvial en el área de relleno, la precipitación máxima mensual, el grado de compactación de los residuos sólidos y el área del sitio para relleno sanitario. Para el dimensionamiento de las celdas diarias se tomó en cuenta la población urbana de la ciudad, la producción diaria de basura, la cobertura y los días destinados a la recolección.

Para el cálculo de la dimensión de la zanja se tomó como referencia las tablas de Jaramillo, 2002, quien establece que para la disposición de residuos sólidos cada zanja debe estar ubicada en la parte inferior del terreno, con una vida útil de 60 días.

\section{RESULTADOS}

Para la evaluación de la ubicación del nuevo relleno sanitario se emplearon los criterios del INIFOM.

En el cuadro 1 se presenta la evaluación de tres posibles localizaciones, quedando seleccionado según la norma, la Comunidad de "La Cruz", sitio número 2.

Para el dimensionamiento de las celdas diarias se tomó en cuenta la población urbana de la ciudad $(94,140$ habitantes), la producción diaria de basura $(0.42 \mathrm{~kg} / \mathrm{hab} /$ día), la cobertura del $87 \%$ y los días de recolección (6 días) obteniéndose las siguientes dimensiones: $1 \mathrm{~m}$ de altura, $2.94 \mathrm{~m}$ de ancho y $2.94 \mathrm{~m}$ de largo, para un volumen de $8.66 \mathrm{~m} 3$.

Por otro lado, el área del terreno es de 250 x 460m, lo que supone un volumen de 115,000 m3 para la disposición de residuos sólidos. Las dimensiones de cada una de las zanjas para el relleno sanitario son $86.46 \mathrm{~m}$ de largo, $4 \mathrm{~m}$ de altura y $9 \mathrm{~m}$ de ancho, lo que da como resultado un total de 46 zanjas con una distancia lateral de un metro entre ellas y una distancia frontal de $6 \mathrm{~m}$ que servirá para el camino de los camiones recolectores. 


\begin{tabular}{|c|c|c|c|c|}
\hline Aspecto & $\begin{array}{c}\text { Nombre del } \\
\text { sitio } \# 1 \\
\text { Relleno Actual }\end{array}$ & $\begin{array}{l}\text { Nombre del } \\
\text { sitio } \# 2 \\
\text { La Cruz }\end{array}$ & $\begin{array}{l}\text { Nombre del } \\
\text { sitio } \# 3 \\
\text { Las Pintadas }\end{array}$ & Norma \\
\hline $\begin{array}{l}\text { 1. Distancia al perimetro de la } \\
\text { ciudad }\end{array}$ & $\sim 5 \mathrm{~km}$ & $\sim 4 \mathrm{~km}$ & $\sim 2,5 \mathrm{~km}$ & $>1,000$ metros \\
\hline $\begin{array}{l}\text { 2. Periodo de traslado al centro } \\
\text { de la ciudad }\end{array}$ & 30 minutos & 20 minutos & 18 minutos & $<30$ minutos \\
\hline $\begin{array}{l}\text { 3. Dirección del crecimiento } \\
\text { de la ciudad }\end{array}$ & Contraria & Contraria & a favor & Contraria al sitio \\
\hline $\begin{array}{l}\text { 4. Distancia a grupos de } \\
\text { viviendas, industrias, escuelas, } \\
\text { hospitales, areas de recreación }\end{array}$ & $4 \mathrm{~km}$ & $3 \mathrm{~km}$ & $1 \mathrm{~km}$ & $>1,000 \mathrm{~m}$ \\
\hline 5. Distancia de via principal & $3 \mathrm{~km}$ & $2 \mathrm{~km}$ & Al borde & $<300 \mathrm{~m}$ \\
\hline $\begin{array}{l}\text { 6. Distancia de linea limitrofe } \\
\text { municipal }\end{array}$ & $20 \mathrm{~km}$ & $20 \mathrm{~km}$ & $15 \mathrm{~km}$ & $<1,000 \mathrm{~m}$ \\
\hline $\begin{array}{l}\text { 7. Pro fundidad delmanto } \\
\text { freatico }\end{array}$ & $50 \mathrm{~m}$ & $45 \mathrm{~m}$ & $30 \mathrm{~m}$ & $\begin{array}{l}>8 \mathrm{~m} \text {, suelo limo- } \\
\text { arenoso } \\
>5 \mathrm{~m} \text { suelo limoso } \\
>3 \mathrm{~m} \text { suelo } \\
\text { arcilloso }\end{array}$ \\
\hline $\begin{array}{l}\text { \$. Distancia de fuentes de agua } \\
\text { destinadas al } \\
\text { abastecimiento.(Pozos) }\end{array}$ & $11 / 2 \mathrm{~km}$ & $1 \mathrm{~km}$ & $500 \mathrm{~m}$ & $>1,000 \mathrm{~m}$ \\
\hline $\begin{array}{l}\text { 9. Distancia de fuentes de agua } \\
\text { superficiales, rios, lagos, mares, } \\
\text { lagunas }\end{array}$ & $5 \mathrm{~km}$ & $4 \mathrm{~km}$ & $280 \mathrm{~m}$ & $>1,000 \mathrm{~m}$ \\
\hline $\begin{array}{l}10 . \text { Ubicación del sitio con } \\
\text { respecto a la dirección del } \\
\text { viento }\end{array}$ & Sotavento & Sotavento & Sotavento & $\begin{array}{l}\text { Sotavento de la } \\
\text { ciudad }\end{array}$ \\
\hline 11. Tipo de suelo & $\begin{array}{l}\text { Franco- } \\
\text { Arcilloso }\end{array}$ & $\begin{array}{l}\text { Franco - } \\
\text { Arcilloso }\end{array}$ & $\begin{array}{l}\text { Franco- } \\
\text { Arcilloso }\end{array}$ & Arcilloso \\
\hline 12. Vocación de suelo & Pastizal & Pastizal & Pastizal & Poco fërtil \\
\hline $\begin{array}{l}\text { 13. Propieda des de material de } \\
\text { cobertura }\end{array}$ & Permeable & Pemeable & Pemeable & Arcilloso \\
\hline $\begin{array}{l}\text { 14. Distancia al banco de } \\
\text { material de cobertura }\end{array}$ & $2,5 \mathrm{~km}$ & $2 \mathrm{~km}$ & $1 \mathrm{~km}$ & $<1,000 \mathrm{~m}$ \\
\hline 15. Vida útil & $>\operatorname{de~} 10$ años. & $>\operatorname{de} 10$ años. & $>\operatorname{de} 10$ años. & $>10$ años \\
\hline $\begin{array}{l}\text { 16. Pendiente y estado de las } \\
\text { vias de acceso al sitio }\end{array}$ & $\begin{array}{l}<10^{\circ}, \text { Buen } \\
\text { estado }\end{array}$ & $\begin{array}{c}<10^{\circ}, \text { Buen } \\
\text { Estado }\end{array}$ & $\begin{array}{l}<25^{\circ}, \text { regular } \\
\text { estado }\end{array}$ & $\begin{array}{l}<10 \text { grados, buen } \\
\text { estado }\end{array}$ \\
\hline 17. Costo del terreno & & & & Opcional \\
\hline 18. Servicio de agua potable & & & & Opcional \\
\hline
\end{tabular}

Cuadro 1. Evaluación de tres posibles ubicaciones del relleno sanitario 
El cálculo de la vida útil del relleno se basó en el área total, un factor de obras adicionales del $30 \%$, el volumen de las zanjas y la capacidad volumétrica del sitio. Con esto la vida útil se proyectó para 20 años.

El caudal medio de lixiviados se calculó de acuerdo a los parámetros establecidos por Jaramillo, 2002 y se presenta en el cuadro 2 .

Cuadro 2. Caudal Medio de Lixiviados calculado para el nuevo relleno

\begin{tabular}{|l|c|}
\hline \multicolumn{1}{|c|}{ Parámetro } & Área del Sitio \\
\hline Precipitación Máxima Mensual (mm/mes) & 850 \\
\hline Area $\left(\mathrm{m}^{2}\right)$ & 115,000 \\
\hline Coeficiente & 0.25 \\
\hline Caudal medio $\left(\mathrm{m}^{3} / \mathrm{mes}\right)$ & $\mathbf{2 4 4 3 7 . 5 0}$ \\
\hline
\end{tabular}

Todos estos resultados permitirán efectuar el estudio económico y ambiental, para el alcance de los objetivos propuestos en esta investigación.

\section{CONCLUSIONES}

En relación a la propuesta de diseño de un relleno: El sitio utilizado como botadero a cielo abierto no puede ser rehabilitado debido a que está saturado de residuos y no posee una vida útil mayor a 5 años.

Debido a la problemática ambiental y riesgo a la salud humana se determinó que el botadero a cielo abierto se debe clausurar con el objetivo de minimizar los impactos ambientales ocasionados.

La celda diaria de residuos sólidos es de $8.66 \mathrm{~m} 3$, con 1 metro de altura, 2.94 metros de ancho y 2.94 metros de largo. En el sitio caben 46 zanjas de $3112.56 \mathrm{~m} 3$ cada una, con las dimensiones de 4 metros de profundidad, 9 metros de ancho y 86.46 metros de largo.

La tierra excavada de las zanjas es de textura arcillosa y sirve como material de cobertura.

Para impermeabilizar el relleno sanitario se propune colocar sobre su superficie una capa de $30 \mathrm{~cm}$ de arcilla compactada mecánicamente.

Los residuos sólidos generan 44,295.62m3/año de lixiviados que pueden ser almacenados en la fosa séptica. Para evitar el aumento de lixiviados se deben construir dos canales de desviación de las aguas de escorrentía.
Se debe construir un sistema de drenaje de gases de piedra grava o bola, de manera simultánea a la construcción de la celda diaria.

Se debe continuar con el estudio para determinar la factibilidad económica y ambiental.

\section{BIBLIOGRAFIA}

INIFOM. (1996): "Estudio Sobre el Sistema de Recolección y Tratamiento de Basura en 41 Municipios de Nicaragua. Diagnostico y Recomendaciones. Municipio de Estelí". Instituto Nicaragüense de Fomento Municipal (INIFOM). Murad Asesores S.A. de C.V. EPACNIC Consultores. Nicaragua.

Jaramillo, J. (2002): “Guía para el Diseño, Construcción y Operación de Rellenos Sanitarios Manuales. Una Solución para la Disposición Final de los Residuos Sólidos Municipales en Pequeñas Poblaciones". Centro Panamericano de Ingeniería Sanitaria y Ciencias del Ambiente (CEPIS). Organización Panamericana de la Salud (OPS). Organización Mundial de la Salud (OMS). Perú.

Lazo, Z. Maltez, F, Ríos, D. (2003): “Caracterización de los Desechos Sólidos y Diseño del Relleno Sanitario de la Ciudad de Diriamba, 2002-2003”. Monografía para Optar al Título de Ingeniero Civil. Universidad Nacional de Ingeniería. Nicaragua.

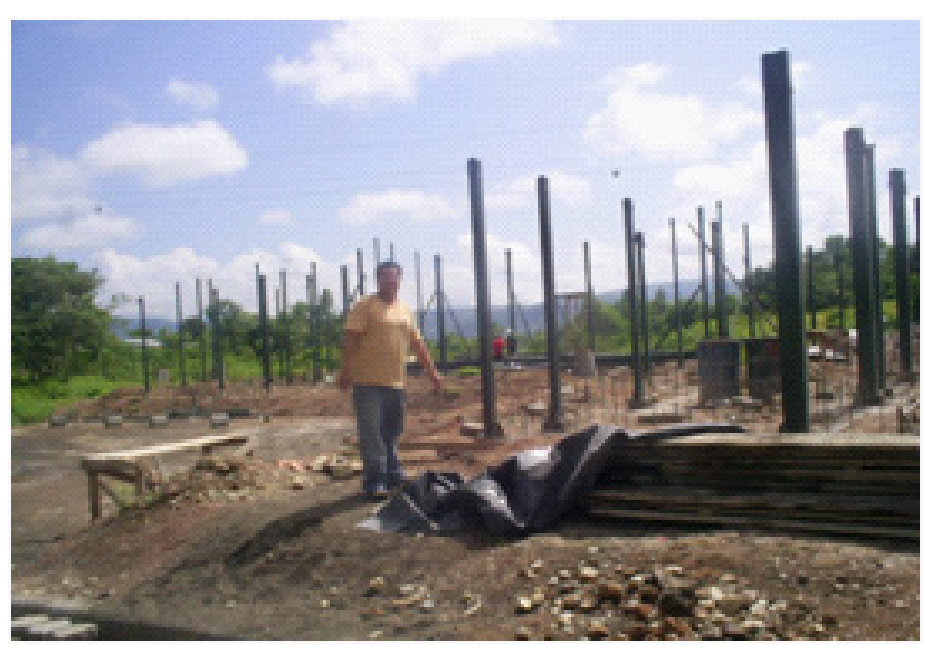

